\title{
Cranial Ultrasound Lesions in the NICU Predict Cerebral Palsy at Age 2 Years in Children Born at Extremely Low Gestational Age
}

\begin{abstract}
Karl C. K. Kuban, MD, SM Epi, Elizabeth N. Allred, MS, T. Michael O'Shea, MD, MPH, Nigel Paneth, MD, MPH, Marcello Pagano, PhD, Olaf Dammann, MD, Alan Leviton, MD, Adré Du Plessis, MBChB, MPH, Sjirk J. Westra, MD, Cindy R. Miller, MD, Haim Bassan, MD, Kalpathy Krishnamoorthy, MD, Joseph Junewick, MD, Nicholas Olomu, MB, BS, Elaine Romano, MSN, Joanna Seibert, MD, Steve Engelke, MD, Padmani Karna, MBBS, Daniel Batton, MD, Sunila E. O'Connor, MD, and Cecelia E. Keller, PT, MHA for the ELGAN study investigators From the Division of Pediatric Neurology, Department of Pediatrics, Boston Medical Center, Boston University, Boston, Massachusetts (KCKK); Neuroepidemiology Unit, Department of Neurology, Children's Hospital Boston, Harvard University, Boston, Massachusetts (ENA, MP, OD, AL); Department of Biostatistics, Harvard School of Public Health, Harvard University, Boston, Massachusetts (ENA, MP); Department of Neonatology, Wake Forest University, Winston-Salem, North Carolina (TMO); Departments of Epidemiology and Pediatrics \& Human Development, College of Human Medicine, Michigan State University, East Lansing, Michigan (NP, NO, PK); Division of Newborn Medicine, Floating Hospital for Children at Tufts Medical Center, Boston, Massachusetts (OD, CEK); Department of Neurology, Children's Hospital Boston, Harvard University, Boston, Massachusetts (ADP, HB); Brighamand Women's Medical Center, Boston, Massachusetts (ADP); Massachusetts General Hospital, Boston, Massachusetts (SJW, KK); Harvard Medical School, Harvard University, Boston, Massachusetts (SJW); Yale University School of Medicine, New Haven, Connecticut (CRM, ER); DeVos Children's Hospital, Grand Rapids, Michigan (JJ); Arkansas Children's Hospital and University of Arkansas Medical Science, Little Rock, Arkansas (JS); East Carolina University, Greenville, North Carolina (SE); William Beaumont Hospital, Royal Oak, Michigan (DB); and Department of Neurology, Section of Pediatric Neurology at the Medical College of Wisconsin, Milwaukee, Wisconsin (SEO).
\end{abstract}

\begin{abstract}
Our prospective cohort study of extremely low gestational age newborns evaluated the association of neonatal head ultrasound abnormalities with cerebral palsy at age 2 years. Cranial ultrasounds in 1053 infants were read with respect to intraventricular hemorrhage, ventriculomegaly, and echolucency, by multiple sonologists. Standardized neurological examinations classified cerebral palsy, and functional impairment was assessed. Forty-four percent with ventriculomegaly and 52\% with echolucency developed cerebral palsy. Compared with no ultrasound abnormalities, children with echolucency were 24 times more likely to have quadriparesis and 29 times more likely to have hemiparesis. Children with ventriculomegaly were 17 times more likely to have quadriparesis or hemiparesis. Forty-three percent of children with cerebral palsy had normal head ultrasound. Focal white matter damage (echolucency) and diffuse damage (late ventriculomegaly) are associated with a high probability of cerebral palsy, especially quadriparesis. Nearly half the cerebral palsy identified at 2 years is not preceded by a neonatal brain ultrasound abnormality.
\end{abstract}

(C) 2009 Sage Publications

Address correspondence to: Karl C. K. Kuban, MD, SM Epi, 1 Boston Medical Center Place, Dowling 3 South, Boston, MA 02118; karl.kuban@bmc.org.

Reprints: http://www.sagepub.com/journalsReprints.nav

The authors have no conflicts of interest to disclose with regard to this article. 


\section{Keywords}

cerebral palsy; extremely low gestational age; cranial ultrasound

Cranial ultrasound studies are used both to identify acute cerebral events in newborns and to assist with prognosis of motor and cognitive dysfunctions. For example, white matter damage, most often identified by a cranial ultrasound abnormality, is the single strongest predictor of cerebral palsy. ${ }^{1-11}$ One limitation of many previous prognostic studies of neonatal ultrasound lesions is the reliance on a single sonologist to interpret scans. Because of the inherent variability in interpreting cranial sonograms, multiple readers may increase reliability. ${ }^{12}$ Another limitation of previous prognostic studies is the lack of replicable operational definitions of cerebral palsy and its types. ${ }^{1-11,13-19}$

In this article, we report how well cranial ultrasound scans obtained in the neonatal intensive care unit predicted cerebral palsy types and severity of motor dysfunction when children were 2 years old, corrected age. Our study of 1053 children born before the 28th postmenstrual week differs from previous studies in several ways. First, the protocol scans of these children were read by at least 2 independent sonologists for congruence about major abnormalities including intraventricular hemorrhage, moderate/severe ventriculomegaly, echogenic lesion, and echolucent lesion. Second, the sonologists' evaluation included specifically information about the location, extent, and laterality of these lesions. Third, the children were given a standardized neurological examination; the standardization of the examination resulted in a low interobserver variability for interpretation of examination findings. ${ }^{20}$ Fourth, those performing the clinical exam were blinded to, and thus not biased by, ultrasound findings. Fifth, we created a cerebral palsy computer-driven diagnostic algorithm, which standardized the diagnosis of topography-based types of cerebral palsy. ${ }^{21}$

\section{Methods}

\section{The ELGAN Study}

The ELGAN study was designed to identify characteristics and exposures that increase the risk of structural and functional neurologic disorders in extremely low gestational age newborns. During the years 2002-2004, women delivering before 28 weeks gestation at 1 of 14 participating institutions in 11 cities in 5 states were asked to enroll in the study. The individual institutional review boards approved the enrollment and consent processes.

Mothers were approached for consent either upon antenatal admission or shortly after delivery, depending on clinical circumstance and institutional preference, and 1249 mothers of 1506 infants consented (Figure 1). Approximately 260 women were either missed or did not consent to participate.

Eighty-eight percent (1053/1198) of eligible children were examined at close to 24 months postterm equivalent.

\section{Imaging Protocol}

Routine ultrasound examinations were performed by technologists at all of the hospitals using digitized high frequency transducers ( 7.5 and $10 \mathrm{MHz}$ or higher). Ultrasound examinations always included as a minimum 6 standard coronal and 5 parasagittal views using the anterior fontanel as the sonographic window. ${ }^{22}$ Mastoid views were not routinely obtained.

Of the 1506 infants enrolled, 1455 had at least 1 protocol ultrasound scan set and, of these, 1053 were examined at 2 years corrected age (Figure 1). The 3 sets of protocol scans were 
defined by the postnatal day on which they were obtained. Protocol 1 scans were obtained between the first and fourth day $(\mathrm{N}=795)$; protocol 2 scans were obtained between the fifth and fourteenth day $(\mathrm{N}=981)$, and protocol 3 scans were obtained between the 15th day and the 40th postconceptual week $(\mathrm{N}=1016) .722 \mathrm{had}$ all 3 sets of ultrasound studies.

\section{Reading Procedures}

Several methods were employed to minimize observer variability. A standard data collection form was developed, which included templates for evaluating ventricular size, parenchymal injury, and intraventricular hemorrhage. A manual with definitions and instructions was distributed. Lastly, a "dry run" review of a series of cases was performed during a conference call to discuss differences in interpretation. ${ }^{12}$

Each ultrasound examination was first read by a study sonologist at the institution of the infant's birth. The images were sent to a sonologist at another ELGAN study institution for a second reading, usually embedded on a compact disc (eFilm Workstation TM, Merge Healthcare/ Merge eMed, Milwaukee, Wisconsin) although occasionally as digitized film. The eFilm program also provided options to adjust and enhance the studies the original reader had done, including the ability to zoom and alter contrast and brightness. Clinical information was not available to either the initial or secondary interpreter.

If there was discrepancy regarding the recognition of intraventricular hemorrhage, moderate/ severe ventriculomegaly, echogenicity, or echolucency, the images were sent to a third (tiebreaking) reader who was blind to what the previous readers reported. Because interobserver variability for echogenic lesions was high ${ }^{12}$ and because transient echogenicities were shown not to be predictive of poor outcome, 23 the following analyses focus on intraventricular hemorrhage, ventriculomegaly, and echolucency.

Sixteen white matter zones in each hemisphere were evaluated for echogenicity and echolucency. The data collection did not require a diagnosis to accompany these lesions, nor were criteria provided for any diagnosis. Rather, the sonologist was free to apply the labels of cystic periventricular leukomalacia, and periventricular hemorrhagic infarction as deemed appropriate, beyond the observations of echogenicity, echolucency, intraventricular hemorrhage, and ventriculomegaly that were mandated for all. Congruence for periventricular leukomalacia and periventricular hemorrhagic infarction diagnosis was not undertaken.

\section{Cerebral Palsy}

The neurological examiners studied a manual, a data collection form and an instructional compact disc designed to minimize examiner variability. Subsequently, they demonstrated acceptably low variability. ${ }^{20}$ The diagnosis of cerebral palsy type (quadriparesis, hemiparesis, and diparesis) was based on an algorithm using these data. ${ }^{21}$

\section{Gross Motor Functional Classification Scale}

The neurological examiners rated each child based on the Gross Motor Function Classification System. All children with a Gross Motor Functional Classification System of 2 or higher had a cerebral palsy diagnosis, and are classified here as having more severe cerebral palsy.

\section{Data Analysis}

The null hypothesis was that no ultrasound lesion predicts cerebral palsy occurrence, cerebral palsy severity, or cerebral palsy type. The primary ultrasound lesions evaluated were intraventricular hemorrhage, ventriculomegaly, and echolucency. The lesion characteristics included location, specifically anterior and posterior locations, extent as measured by the number of anatomic locations involved, unilateral or bilateral involvement, and identification 
of what sonologists called periventricular hemorrhagic infarction and periventricular leukomalacia. Cerebral palsy types included quadriparesis, hemiparesis, and diparesis, and cerebral palsy severity was determined according to Gross Motor Functional Classification System categorization.

We present row percents in most tables to show the proportion of children with a particular ultrasound lesion who had each type of cerebral palsy and the associated degree of impairment. Using cerebral palsy type and severity as the outcomes of interest, we evaluated the sensitivities, specificities, and predictive values of ventriculomegaly and echolucency seen on head ultrasound study in the neonatal period.

\section{Results}

\section{Overview (Table 1-Table 3)}

Eleven and a half percent of all children in our sample were given a diagnosis of cerebral palsy at approximately 2 years corrected age. The risk of cerebral palsy was $9 \%$ if the scan had isolated intraventricular hemorrhage, similar in prevalence to that observed among children with completely normal head ultrasound studied (6\%). Forty-three percent (51/120) of children with cerebral palsy had normal cranial ultrasound studies (Table 1 and Table 2). In contrast, $34 \%$ of those whose scan had isolated echolucency in the cerebral white matter and $39 \%$ of those whose scan had isolated ventriculomegaly developed cerebral palsy (Table 1).

Ultrasound lesions, however, tend not to occur in isolation. For example, $87 \%$ of scans with ventriculomegaly and $62 \%$ of scans with intraventricular hemorrhage had an additional lesion (Table 2).

Among children who had ventriculomegaly or echolucency, the risk of quadriparesis was 3 to 5 times higher than the risk of diparesis. In contrast, among those with no head ultrasound study abnormality, the risk of quadriparesis and diparesis were comparable (Table 1). Viewed differently, 34\% (22/64) of children who developed quadriparesis and 60\% (22/37) of children who developed diparesis did not have intraventricular hemorrhage, echolucency, or ventriculomegaly (Table 2). Nearly half of those with cerebral palsy had a Gross Motor Functional Classification System of 2 or greater, and $85 \%$ of these children had quadriparesis.

\section{Hemorrhage (Table 3)}

Nine percent of children who had a unilateral intraventricular hemorrhage and $28 \%$ of children who had bilateral intraventricular hemorrhage developed cerebral palsy. Also, blood in the third and fourth ventricles, a correlate of ventriculomegaly, predicted quadriparesis better than blood in the lateral ventricle. Of the 10 children who had bilateral cerebellar hemorrhages, 3 developed quadriparesis and 3 developed hemiparesis.

\section{Moderate/Severe Ventriculomegaly (Table 4)}

Early studies (examinations performed during the first 14 postnatal days)—Of children who had unilateral ventriculomegaly, $21 \%$ developed hemiparesis and $7 \%$ developed quadriparesis. In contrast, those who had bilateral ventriculomegaly were more likely to develop quadriparesis than hemiparesis (23\% versus 6\%). Diparesis occurred in 13\% to $14 \%$ of children with ventriculomegaly, whether unilateral or bilateral.

Late studies (examinations performed between postnatal day 15and expected date of confinement)-Ventriculomegaly on the third protocol scan was a better predictor of cerebral palsy than ventriculomegaly on an early scan. For example, the rate of cerebral palsy among the 19 subjects who had early ventriculomegaly, but never had late ventriculomegaly, was $21 \%$ (5\% with quadriparesis, $5 \%$ with hemiparesis, and $11 \%$ with 
diparesis; data not shown), compared to $50 \%$ of children with ventriculomegaly on a late scan. Unlike early unilateral ventriculomegaly, late unilateral ventriculomegaly predicted quadriparesis even better than it predicted hemiparesis. Nearly half of those with bilateral late ventriculomegaly had the quadriparetic form of cerebral palsy and a third had more severe impairment (Gross Motor Functional Classification System >2). Diparesis occurred in less than $8 \%$ of cases among those with late ventriculomegaly regardless of location or laterality of the ventricular enlargement.

\section{Laterality of Echolucent Lesions and Empiric Diagnoses}

Seventy-one children had an echolucency on head ultrasound study, and one third with a unilateral lesion and two thirds with bilateral lesions developed cerebral palsy. Unilateral lesions predicted similar rates of quadriparesis and hemiparesis (17\% and 15\%), whereas bilateral lesions were 7 to 13 times more likely to predict quadriparesis. Diparesis occurred in only $4 \%$ of those with either unilateral or bilateral echolucency.

Approximately $45 \%$ of children whose scan was given a diagnosis of cystic periventricular leukomalacia or periventricular hemorrhagic infarction developed cerebral palsy, which, in over $60 \%$ of cases, was classified as quadriparesis. Less than a third of those with cerebral palsy and periventricular hemorrhagic infarction, which is a predominantly unilateral lesion, had hemiparesis. Fewer yet with either periventricular hemorrhagic infarction (19\%) or cystic periventricular leukomalacia (28\%) had Gross Motor Functional Classification System level of 2 or greater.

\section{Extent of Echolucent Lesions}

Each hemisphere was divided into 16 zones on the ultrasound data collection form. Although no linear trend was seen between the number of zones involved and the risk of cerebral palsy, cerebral palsy type or severity, $85 \%$ of the 13 children whose scan had 5 or more echolucency zones developed cerebral palsy.

\section{Location of Echolucent Lesions}

Only 5 children with diparesis had echolucency and none of the lesions were seen in posterior regions. The echolucency lesions seen in hemiparetics and quadriparetics were seen equally frequently in the anterior and posterior aspects of the cerebral hemispheres. Those with hemiparesis were more than twice as likely to have unilateral lesions as bilateral lesions, whereas those with quadriparetics were more than 3 times more likely to have bilateral lesions as unilateral lesions (data not shown).

Infants deemed to have cystic periventricular leukomalacia were as likely to have echolucency lesions placed anteriorly as placed posteriorly. Among scans with periventricular hemorrhagic infarction, echolucency lesions were more likely to occur anteriorly. More than half of scans with periventricular hemorrhagic infarction also had lesions along the more posteriorly placed body of the lateral ventricles.

\section{Ultrasound Lesions as Predictors of Cerebral Palsy (Table 5 and Table 6)}

Echolucency, ventriculomegaly, and intraventricular hemorrhage were strongly and significantly associated with quadriparesis (risk ratio: $24,17,5.1$ ) and hemiparesis (risk ratio: 29,17 , and 5.8). Diparesis also was significantly associated with all 3 head ultrasound study findings, but less strongly (risk ratio: 5, 5.7, and 2.3).

Among infants with ventriculomegaly, $44 \%$ developed cerebral palsy, and among infants with echolucency, 52\% developed cerebral palsy (positive predictive value). Ventriculomegaly and echolucency predicted more severe cerebral palsy (Gross Motor Functional Classification 
System of 2 or greater) and quadriparesis in approximately half of children with cerebral palsy. For quadriparesis and hemiparesis, the sensitivity of ventriculomegaly and echolucency ranged from 38 to $47 \%$, whereas for diparesis it was 14 and $24 \%$. All negative predictive values and specificities were $92 \%$ or higher.

\section{Discussion}

\section{Overview}

Based on our knowledge, this is the largest study to date relating neonatal cranial ultrasound findings and cerebral palsy at 2 years in extremely low gestational age neonates. We reject the null hypothesis that specific lesions do not predict specific types or severity of cerebral palsy. In general, the primary finding reported here is that about half the children with ventriculomegaly or echolucency developed cerebral palsy, and half of these children developed more severe cerebral palsy. Additionally, almost half of all children with cerebral palsy had an entirely normal head ultrasound study. Children with isolated intraventricular hemorrhage were only slightly more likely to have cerebral palsy than those with a normal head ultrasound study. Late occurrence of ventriculomegaly, bilateral echolucency, and identification of periventricular hemorrhagic infarction or periventricular leukomalacia were particularly predictive of quadriparesis. In contrast to children with normal head ultrasound study whose risk of quadriparesis and diparesis were comparable, children with abnormal head ultrasound study findings were 3 to 5 times more likely to have quadriparesis than diparesis. Finally, a diagnosis of periventricular hemorrhagic infarction was twice as likely as a diagnosis of cystic periventricular leukomalacia to predict hemiparesis, while cystic periventricular leukomalacia was twice as likely to predict quadriparesis as hemiparesis.

\section{Head Ultrasound-Cerebral Palsy Associations}

Hemorrhage-Isolated intraventricular hemorrhage or echogenic lesions predicted cerebral palsy only slightly better than scans without any abnormality, an observation made previously. $19,24,25$ Yet, over a third of the infants with intraventricular hemorrhage developed cerebral palsy, which we attribute to the presence of associated echolucency and/or ventriculomegaly, indicators of white matter damage ${ }^{26}$ and independent predictors of cerebral palsy. Of the 10 infants with bilateral cerebellar hemorrhage, 6 had cerebral palsy, an association reported previously. ${ }^{27-30}$ Cerebellar hemorrhage was identified in a total of 14 infants, which may be an underestimate of true prevalence because mastoid views were not routinely obtained.

Severity-Overall, nearly half the children with cerebral palsy were more severely affected, defined by having a Gross Motor Functional Classification System of 2 or greater, comparable to other studies looking at cohorts born at comparable gestational age. ${ }^{31,32}$ Nearly all children with more severe cerebral palsy had quadriparesis and most children with quadriparesis had more severe cerebral palsy. Compared to those without head ultrasound study abnormalities, children who had either ventriculomegaly or echolucency were 11 to 16 times more likely to have a Gross Motor Functional Classification System grade of 2 or greater and 17 to 24 times more likely to have quadriparesis. Those with diparesis were least likely to be more severely involved, also comparable to findings by others. ${ }^{32}$ The importance of distinguishing those with quadriparesis and/or more severe forms of cerebral palsy is underscored by the observation in our cohort that these children have a markedly elevated risk of having microcephaly and cognitive impairment and for screening positive on the Modified Checklist for Autism in Toddlers. ${ }^{21}$

As others have shown, the presence of more than 1 abnormal ultrasound feature generally increased the risk of developing quadriparesis or more severe forms of cerebral palsy. ${ }^{5}$ On the 
other hand, once an echolucency was identified in our cohort, the risk of more severe cerebral palsy was not increased with additional abnormal ultrasound features.

Echolucent lesions and ventriculomegaly-Our findings that echolucency and ventriculomegaly are associated with subsequent cerebral palsy and that about one half of children with cerebral palsy have one or both of these abnormalities, are comparable to the findings of others. ${ }^{9,11,19,31,33-37}$ Compared to children with no head ultrasound study abnormalities, children who had echolucency were 24 times more likely to have quadriparesis, 29 times more likely to have hemiparesis, and 5 times more likely to have diparesis. The advantages of our study include late imaging for 1016 of 1053 subjects, agreement about abnormalities by 2 or 3 independent readers of ultrasound studies by individuals and had no knowledge of the clinical history, and implementation of a standardized method of identifying cerebral palsy.

Compared to children with no head ultrasound study abnormalities, children who had ventriculomegaly were 17 times more likely to have quadriparesis and hemiparesis and nearly 6 times more likely to have diparesis. Ventriculomegaly identified closer to term appears to be more predictive of cerebral palsy than the presence of ventriculomegaly seen within the first 2 weeks of life. Conversely, those who had ventriculomegaly early, but did not demonstrate ventriculomegaly on late scans had rates of quadriparesis that were comparable to those without ventriculomegaly. The importance of late ventriculomegaly as a predictor of later disability was emphasized by Ment, who reported a 45\% risk for cerebral palsy (and a 55\% risk for mental retardation) among those that had ventriculomegaly at term. ${ }^{13}$ This observation may relate to lost white matter volume as a contributor to late ventriculomegaly in contradistinction to transient, early ventricular enlargement due to the presence of intraventricular blood.

Cerebral palsy in children who have no head ultrasound study abnormality-Six percent of children whose scan was entirely normal developed cerebral palsy accounting for nearly half of those with cerebral palsy. Others have also shown that among extremely low birth weight infants, those with normal cranial ultrasound studies still carry a risk as high as $9 \%$ for cerebral palsy. ${ }^{14,16}$

Children who develop cerebral palsy and have no abnormality on any ultrasound scan of the head might well have lesions below sonographic resolution, ${ }^{36,38-41}$ an explanation that has been called the "tip of the iceberg" hypothesis. ${ }^{42}$ Some of these may first become evident on ultrasound studies performed near term, as the brain grows, ${ }^{36,39,43-46}$ while some can be identified on routine or specialized magnetic resonance imaging studies, such as diffusion tensor imaging and volumetric evaluation. ${ }^{41,47-49}$ Studies of infants using both ultrasound and magnetic resonance imaging ${ }^{40,50}$ or ultrasound and post-mortem examination, ${ }^{32}$ show that white matter damage identified on sonograms is a relatively small proportion of the damage evident on magnetic resonance or by pathology.

\section{Periventricular hemorrhagic infarction and periventricular leukomalacia-The}

name periventricular hemorrhagic infarction is applied to largely unilateral or very asymmetric lesions. ${ }^{51}$ Indeed, children who had periventricular hemorrhagic infarction were twice as likely to have hemiparesis as quadriparesis, which affirms previous reports by Bassan et al. ${ }^{52}$ Those with hemiparesis were more likely to have unilateral ventriculomegaly or periventricular hemorrhagic infarction than other lesions on head ultrasound study. Also, $60 \%$ of those with periventricular hemorrhagic infarction had bilateral disease manifesting as diparesis or quadriparesis, a finding also noted by Bassan et al. ${ }^{52}$

In contrast to periventricular hemorrhagic infarction, periventricular leukomalacia is more often associated with diparesis. ${ }^{51,53}$ Yet, those deemed to have cystic periventricular 
leukomalacia in this cohort were more than twice as likely to have quadriparesis and hemiparesis as diparesis.

\section{Limitations and Strengths of the Study}

Limitations of the study: head ultrasound study and cranial magnetic resonance imaging findings-Perhaps the main limitation of this study is our dependence on ultrasound to identify white matter damage. Magnetic resonance imaging studies are more sensitive, especially of diffuse white matter damage. ${ }^{36,37}$ In cohorts of infants born at less than 30 weeks gestation, magnetic resonance imaging sensitivity 36,37 and positive predictive value ${ }^{36}$ are more than double that reported using head ultrasound study. Thus, our study might misclassify some infants as not having white matter damage, when, indeed, a magnetic resonance imaging would identify such damage. Mastoid views were not routinely employed, which may lead to an underestimation of cerebellar lesions.

Strengths of the study-Strengths of the current study include the large sample ${ }^{54}$ based on gestational age, ${ }^{55}$ minimized interobserver disagreements about ultrasound findings, ${ }^{12}$ standardized diagnosis of cerebral palsy, ${ }^{21}$ minimized clinical bias by examiners, and the use of high frequency ultrasounds, which were obtained in most after the first month of life, usually close to term, when white matter damage might be seen for the first time in infants with previously normal scans. ${ }^{9}$

\section{Conclusions and Inferences}

Echolucency or ventriculomegaly on head ultrasound study studies are the strongest newborn predictor of subsequent cerebral palsy, especially quadriparesis and more severe cerebral palsy. Yet, nearly half of children who are destined to have cerebral palsy, like many extremely low gestational age newborns who have substantial development disorders, do not have an abnormality on neonatal head ultrasound study studies. These children with cerebral palsy and no recognized head ultrasound study abnormality are at lower risk for quadriparesis and more severe forms of cerebral palsy than children with ventriculomegaly or echolucency. Some, but not all, infants destined to develop cerebral palsy who had normal head ultrasound study have morphologic abnormalities seen on magnetic resonance imaging performed near term. It is unsettled whether magnetic resonance imaging would have been abnormal sooner after birth and whether there is an on-going process that accounts for further changes later that may be amenable to prevention or interruption. Although awaiting a more sensitive method for early identification of extremely low gestational age newborns who will develop cerebral palsy, clinicians should routinely assess these infants' neuromotor status after discharge from intensive care.

\section{Acknowledgments}

Financial support for this research was provided by the National Institute of Neurological Disorders and Stroke.

\section{References}

1. Holling EE, Leviton A. Characteristics of cranial ultrasound white-matter echolucencies that predict disability: a review. Dev Med Child Neurol 1999;41:136-139. [PubMed: 10075101]

2. Ancel PY, Livinec F, Larroque B, et al. Cerebral palsy among very preterm children in relation to gestational age and neonatal ultrasound abnormalities: the EPIPAGE cohort study. Pediatrics 2006;117:828-835. [PubMed: 16510664]

3. Kristjansdottir R, Uvebrant P, Wiklund LM. Clinical characteristics of children with cerebral white matter abnormalities. Eur J Paediatr Neurol 2000;4:17-26. [PubMed: 10701100] 
4. Roelants-van Rijn AM, Groenendaal F, Beek FJ, et al. Parenchymal brain injury in the preterm infant: comparison of cranial ultrasound, MRI and neurodevelopmental outcome. Neuropediatrics 2001;32:80-89. [PubMed: 11414648]

5. Aziz K, Vickar DB, Sauve RS, et al. Province-based study of neurologic disability of children weighing 500 through 1249 grams at birth in relation to neonatal cerebral ultrasound findings. Pediatrics 1995;95:837-844. [PubMed: 7761206]

6. Msall ME, Buck GM, Rogers BT, et al. Risk factors for major neurodevelopmental impairments and need for special education resources in extremely premature infants. J Pediatr 1991;119:606-614. [PubMed: 1919894]

7. Graziani LG, Pasto M, Stanley C, et al. Neonatal neurosonographic correlates of cerebral palsy in preterm infants. Pediatrics 1986;78:88-95. [PubMed: 3523417]

8. Wilson-Costello D, Borawski E, Friedman H, et al. Perinatal correlates of cerebral palsy and other neurologic impairment among very low birth weight children. Pediatrics 1998;102:315-322. [PubMed: 9685432]

9. De Vries LS, Van Haastert IL, Rademaker KJ, et al. Ultrasound abnormalities preceding cerebral palsy in high-risk preterm infants. J Pediatr 2004;144:815-820. [PubMed: 15192633]

10. Cooke RWI. Trends in incidence of cranial ultrasound lesions and cerebral palsy in very low birthweight infants 1982-93. Arch Dis Child Fetal Neonatal Ed 1999;80:F115-F117. [PubMed: 10325787]

11. O'Shea TM, Klinepeter KL, Dillard RG. Prenatal events and the risk of cerebral palsy in very low birth weight infants. Am J Epidemiol 1998;147:362-369. [PubMed: 9508103]

12. Kuban K, Adler I, Allred EN, et al. Observer variability assessing US scans of the preterm brain: the ELGAN study. Pediatr Radiol 2007;37:1201-1208. [PubMed: 17901950]

13. Ment LR, Vohr B, Allan W, et al. The etiology and outcome of cerebral ventriculomegaly at term in very low birth weight preterm infants. Pediatrics 1999;104(2 Pt 1):243-248. [PubMed: 10429002]

14. Sampath V, Bowen J, Gibson F. Risk factors for adverse neurodevelopment in extremely low birth weight infants with normal neonatal cranial ultrasound. J Perinatol 2005;25:210-215. [PubMed: 15578032]

15. Shankaran S, Johnson Y, Langer JC, et al. Outcome of extremely-low-birth-weight infants at highest risk: gestational age $<$ or $=24$ weeks, birth weight $<$ or $=750 \mathrm{~g}$, and 1-minute Apgar $<$ or $=3$. Am J Obstet Gynecol 2004;191:1084-1091. [PubMed: 15507925]

16. Laptook AR, O'Shea TM, Shankaran S, Bhaskar B. Adverse neurodevelopmental outcomes among extremely low birth weight infants with a normal head ultrasound: prevalence and antecedents. Pediatrics 2005;115:673-680. [PubMed: 15741371]

17. Wilson-Costello D, Friedman H, Minich N, et al. Improved neurodevelopmental outcomes for extremely low birth weight infants in 2000-2002. Pediatrics 2007;119:37-45. [PubMed: 17200269]

18. Doyle LW, Betheras FR, Ford GW, et al. Survival, cranial ultrasound and cerebral palsy in very low birthweight infants: 1980s versus 1990s. J Paediatr Child Health 2000;36:7-12. [PubMed: 10723683]

19. Pinto-Martin J, Riolo S, Cnaan A, et al. Cranial ultrasound prediction of disabling and nondisabling cerebral palsy at age two in a low birth weight population. Pediatrics 1995;95:249-254. [PubMed: 7838643]

20. Kuban K, O'Shea M, Allred E, et al. Video and CD-ROM as a training tool for performing neurologic examinations of 1-year-old children in a multicenter epidemiologic study. J Child Neurol 2005;20:829-831. [PubMed: 16417880]

21. Kuban K, Allred EN, O'Shea TM, et al. An algorithm for identifying and classifying cerebral palsy in young children. J Pediatr. 2008 In Press.

22. Teele, R.; Share, J. Ultrasonography of infants and children. Philadelphia: Saunders; 1991.

23. Pisani F, Leali L, Moretti S, et al. Transient periventricular echodensities in preterms and neurodevelopmental outcome. J Child Neurol 2006;21:230-235. [PubMed: 16901425]

24. Paneth, N.; Rudelli, R.; Kazam, E.; Monte, W. Clinics in Developmental Medicine. Vol. vol. 131. London: Mac Keith; 1994. Brain Damage in the Preterm Infant; p. 131

25. Krishnamoorthy KS, Kuban KC, Leviton A, et al. Periventricular-intraventricular hemorrhage, sonographic localization, phenobarbital, and motor abnormalities in low birth weight infants. Pediatrics 1990;85:1027-1033. [PubMed: 2187174] 
26. Kuban K, Sanocka U, Leviton A, et al. White matter disorders of prematurity: association with intraventricular hemorrhage and ventriculomegaly. J Pediatr 1999;134:539-546. [PubMed: 10228286]

27. Johnsen SD, Tarby TJ, Lewis KS, et al. Cerebellar infarction: an unrecognized complication of very low birthweight. J Child Neurol 2002;17:320-324. [PubMed: 12150576]

28. Bodensteiner JB, Johnsen SD. Cerebellar injury in the extremely premature infant: newly recognized but relatively common outcome. J Child Neurol 2005;20:139-142. [PubMed: 15794181]

29. Johnsen SD, Bodensteiner JB, Lotze TE. Frequency and nature of cerebellar injury in the extremely premature survivor with cerebral palsy. J Child Neurol 2005;20:60-64. [PubMed: 15791925]

30. Limperopoulos C, Bassan H, Gauvreau K, et al. Does cerebellar injury in premature infants contribute to the high prevalence of long-term cognitive, learning, and behavioral disability in survivors? Pediatrics 2007;120:584-593. [PubMed: 17766532]

31. Vollmer B, Roth S, Baudin J, et al. Predictors of long-term outcome in very preterm infants: gestational age versus neonatal cranial ultrasound. Pediatrics 2003;112:1108-1114. [PubMed: 14595054]

32. Vohr BR, Msall ME, Wilson D, et al. Spectrum of gross motor function in extremely low birth weight children with cerebral palsy at 18 months of age. Pediatrics 2005;116:123-129. [PubMed: 15995042]

33. Wheater M, Rennie JM. Perinatal infection is an important risk factor for cerebral palsy in very-lowbirthweight infants. Dev Med Child Neurol 2000;42:364-367. [PubMed: 10875520]

34. Nelson KB, Grether JK, Dambrosia JM, et al. Neonatal cytokines and cerebral palsy in very preterm infants. Pediatr Res 2003;53:600-607. [PubMed: 12612192]

35. Neil JJ, Inder TE. Imaging perinatal brain injury in premature infants. Semin Perinatol 2004;28:433443. [PubMed: 15693400]

36. Mirmiran M, Barnes PD, Keller K, et al. Neonatal brain magnetic resonance imaging before discharge is better than serial cranial ultrasound in predicting cerebral palsy in very low birth weight preterm infants. Pediatrics 2004;114:992-998. [PubMed: 15466096]

37. Woodward LJ, Anderson PJ, Austin NC, et al. Neonatal MRI to predict neurodevelopmental outcomes in preterm infants. N Engl J Med 2006;355:685-694. [PubMed: 16914704]

38. Miller SP, Cozzio CC, Goldstein RB, et al. Comparing the diagnosis of white matter injury in premature newborns with serial MR imaging and transfontanel ultrasonography findings. AJNR Am J Neuroradiol 2003;24:1661-1669. [PubMed: 13679289]

39. Maalouf EF, Duggan PJ, Rutherford MA, et al. Magnetic resonance imaging of the brain in a cohort of extremely preterm infants. J Pediatr 1999;135:351-357. [PubMed: 10484802]

40. Inder TE, Anderson NJ, Spencer C, et al. White matter injury in the premature infant: a comparison between serial cranial sonographic and MR findings at term. AJNR Am J Neuroradiol 2003;24:805809. [PubMed: 12748075]

41. Inder TE, Wells SJ, Mogridge NB, et al. Defining the nature of the cerebral abnormalities in the premature infant: a qualitative magnetic resonance imaging study. J Pediatr 2003;143:171-179. [PubMed: 12970628]

42. Leviton A, Gilles F. Ventriculomegaly, delayed myelination, white matter hypoplasia, and "periventricular" leukomalacia: how are they related? Pediatr Neurol 1996;15:127-136. [PubMed: 8888047]

43. Peterson BS, Anderson AW, Ehrenkranz R, et al. Regional brain volumes and their later neurodevelopmental correlates in term and preterm infants. Pediatrics 2003;111(5 Pt 1):939-948. [PubMed: 12728069]

44. Huppi PS, Murphy B, Maier SE, et al. Microstructural brain development after perinatal cerebral white matter injury assessed by diffusion tensor magnetic resonance imaging. Pediatrics 2001;107:455-460. [PubMed: 11230582]

45. Inder TE, Warfield SK, Wang H, et al. Abnormal cerebral structure is present at term in premature infants. Pediatrics 2005;115:286-294. [PubMed: 15687434]

46. Dyet LE, Kennea N, Counsell SJ, et al. Natural history of brain lesions in extremely preterm infants studied with serial magnetic resonance imaging from birth and neurodevelopmental assessment. Pediatrics 2006;118:536-548. [PubMed: 16882805]

47. Abernethy LJ, Palaniappan M, Cooke RW. Quantitative magnetic resonance imaging of the brain in survivors of very low birth weight. Arch Dis Child 2002;87:279-283. [PubMed: 12243993] 
48. Limperopoulos C, Soul JS, Haidar H, et al. Impaired trophic interactions between the cerebellum and the cerebrum among preterm infants. Pediatrics 2005;116:844-850. [PubMed: 16199692]

49. Skranes JS, Vik T, Nilsen G, et al. Cerebral magnetic resonance imaging (MRI) and mental and motor function of very low birth weight infants at one year of corrected age. Neuropediatrics 1993;24:256262. [PubMed: 8309514]

50. Maalouf EF, Duggan PJ, Counsell SJ, et al. Comparison of findings on cranial ultrasound and magnetic resonance imaging in preterm infants. Pediatrics 2001;107:719-727. [PubMed: 11335750]

51. Bassan H, Benson CB, Limperopoulos C, et al. Ultrasonographic features and severity scoring of periventricular hemorrhagic infarction in relation to risk factors and outcome. Pediatrics 2006;117:2111-2118. [PubMed: 16740854]

52. Bassan H, Limperopoulos C, Visconti K, et al. Neurodevelopmental outcome in survivors of periventricular hemorrhagic infarction. Pediatrics 2007;120:785-792. [PubMed: 17908766]

53. Bax M, Tydeman C, Flodmark O. Clinical and MRI correlates of cerebral palsy: the European Cerebral Palsy Study. JAMA 2006;296:1602-1608. [PubMed: 17018805]

54. Kiely JL, Paneth N. Follow-up studies of low-birthweight infants: suggestions for design, analysis and reporting. Dev Med Child Neurol 1981;23:96-100. [PubMed: 6451466]

55. Arnold CC, Kramer MS, Hobbs CA, et al. Very low birth weight: a problematic cohort for epidemiologic studies of very small or immature neonates. Am J Epidemiol 1991;134:604-613. [PubMed: 1951265] 


\section{Enrolled}

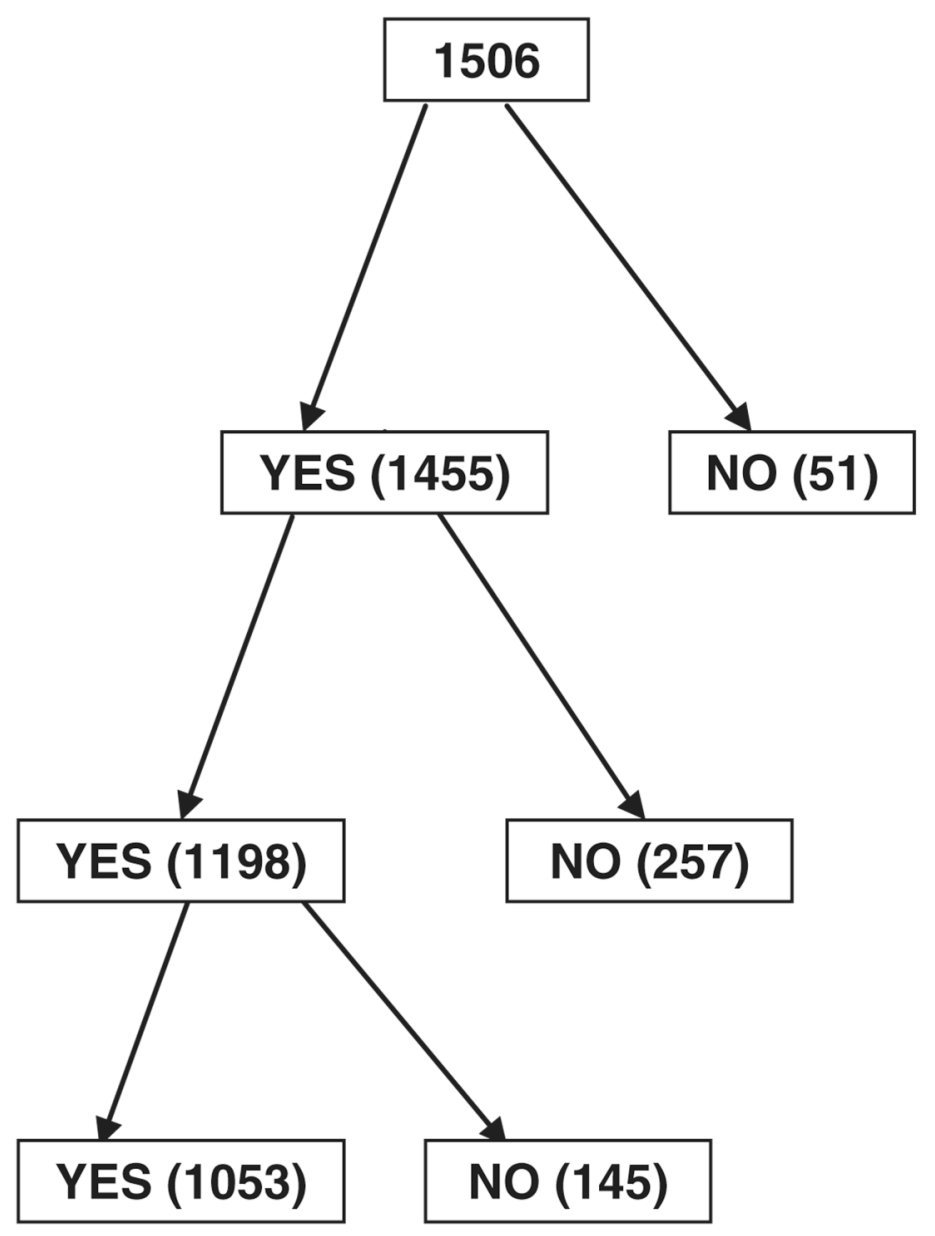

Survived to 24 months

Qualifying head ultrasound study

Examined (Cerebral palsy and gross motor functional classification system) at 24 months

Figure 1.

Sample Description. 


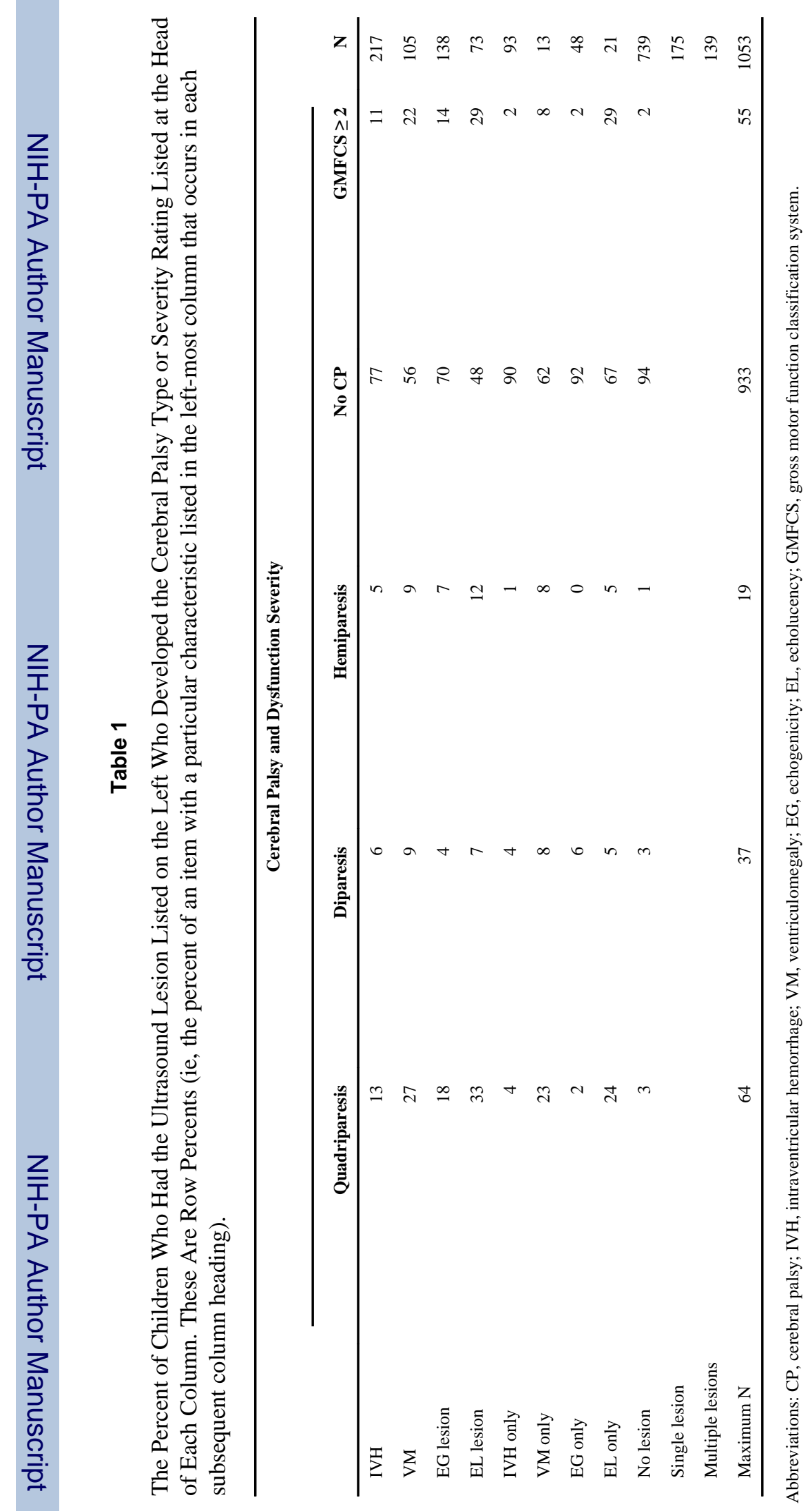

J Child Neurol. Author manuscript; available in PMC 2010 February 1. 


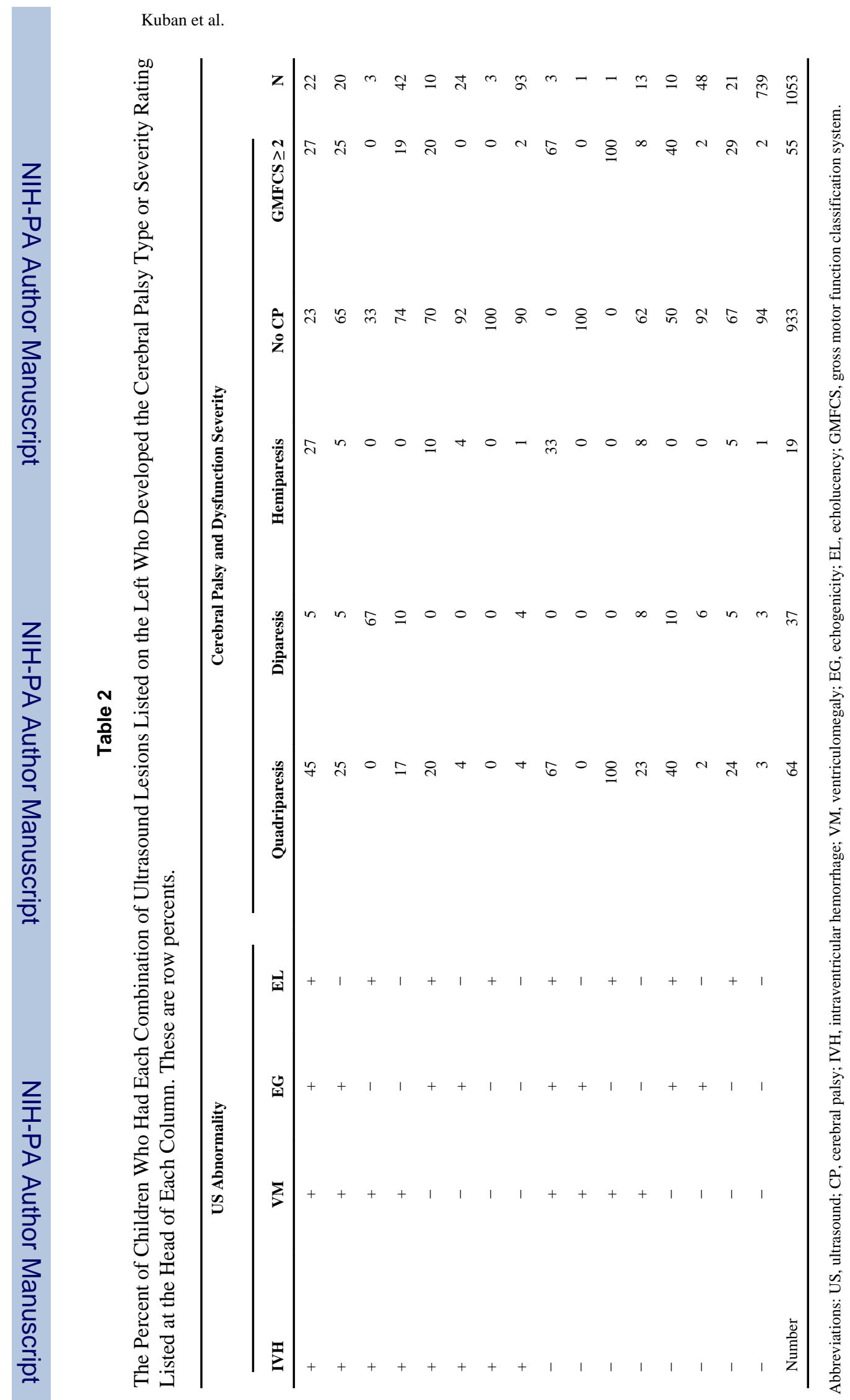

Page 14 


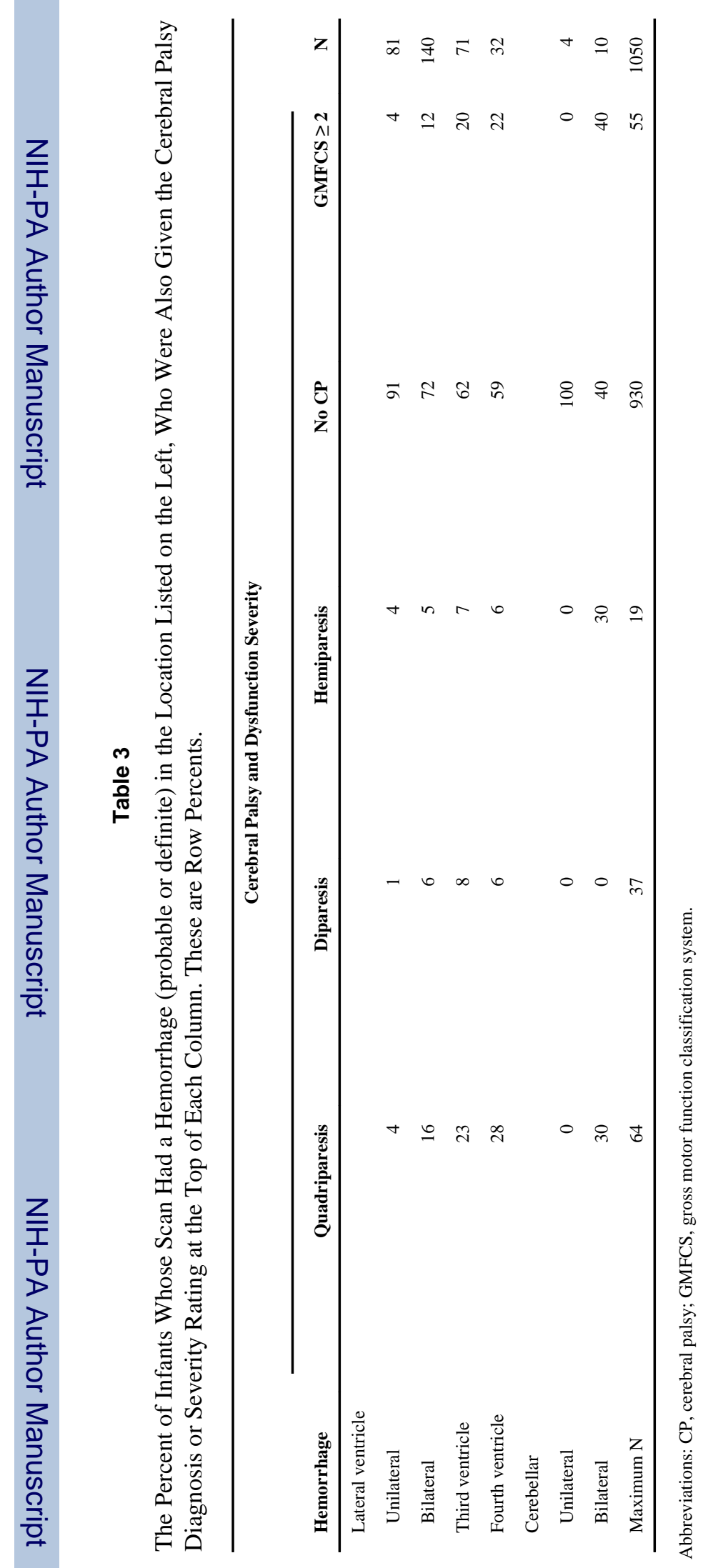

J Child Neurol. Author manuscript; available in PMC 2010 February 1. 


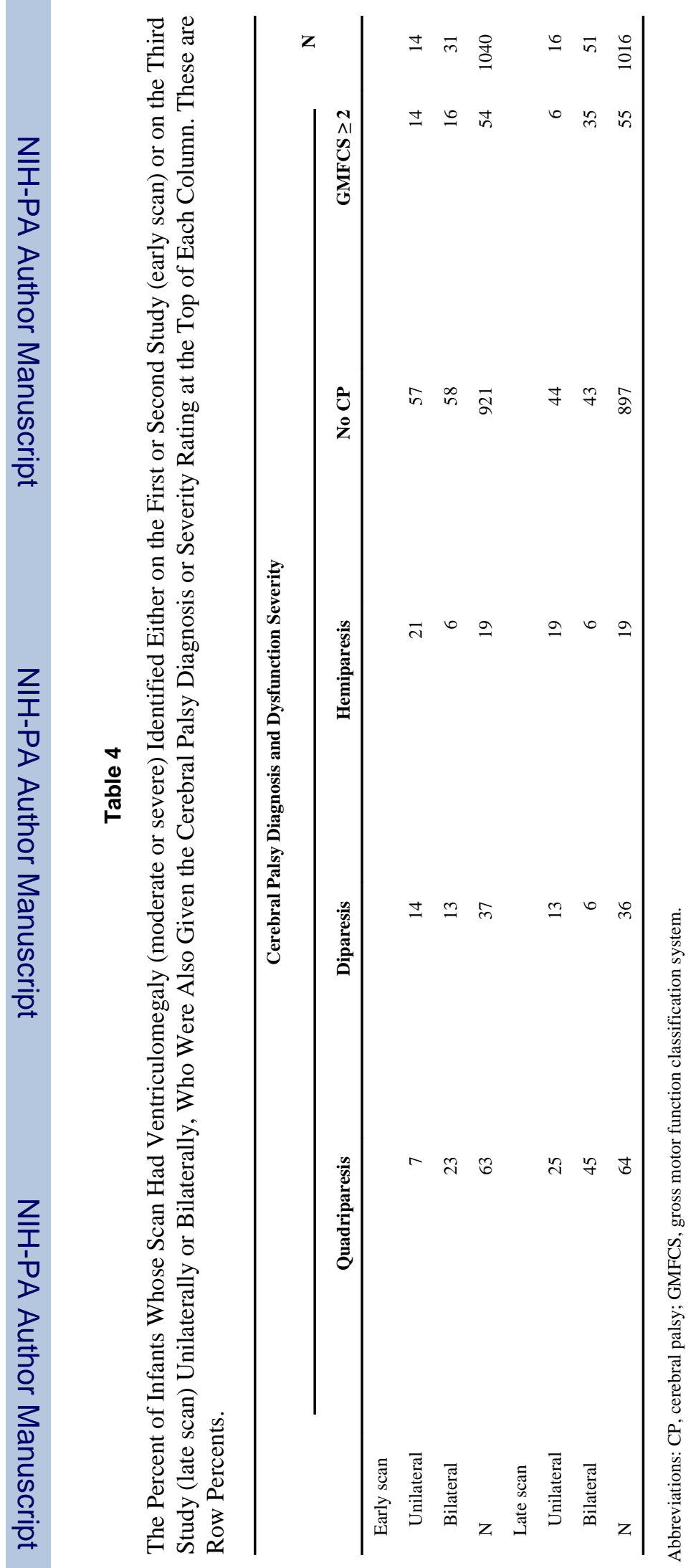

J Child Neurol. Author manuscript; available in PMC 2010 February 1. 


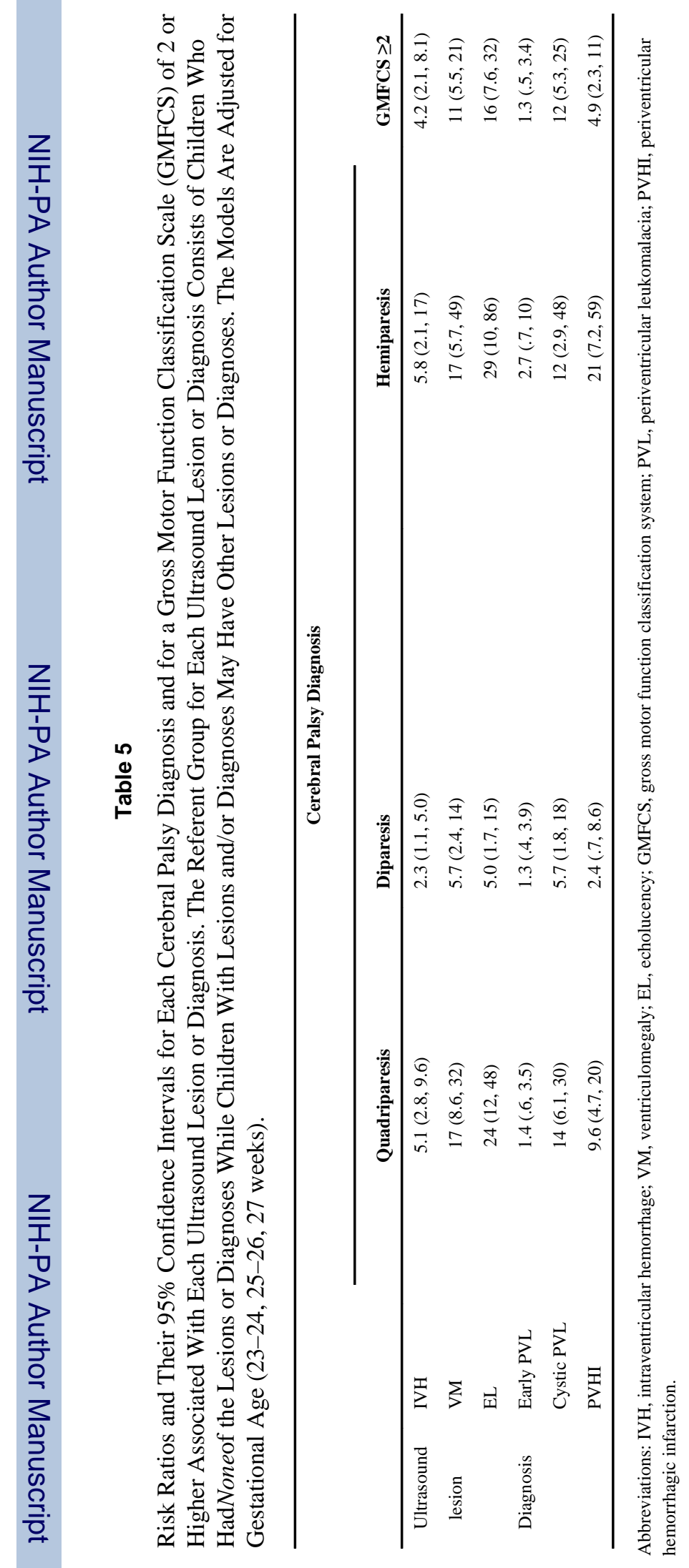

J Child Neurol. Author manuscript; available in PMC 2010 February 1. 


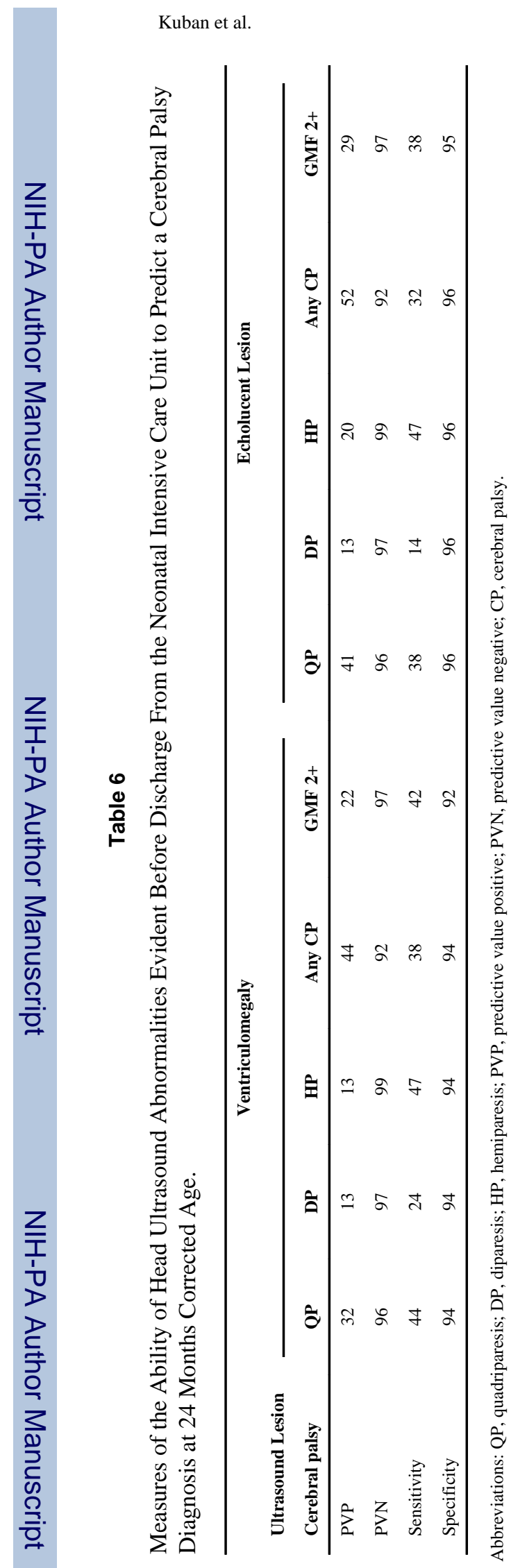

J Child Neurol. Author manuscript; available in PMC 2010 February 1. 